\title{
A Retrospective Mapping of Pelvic Lymph Node Metastasis Distribution in Patients with Endometrial Carcinoma
}

\author{
Ricardo Pedrini Cruz $^{1}$ (D) \\ Received: 6 August 2020 / Accepted: 15 August 2020/Published online: 21 August 2020 \\ (C) Association of Surgeons of India 2020
}

The article by $\mathrm{Hu}$ and colleagues [1] presents a retrospective cohort to analyze metastasis distribution of pelvic lymph node of 216 patients with endometrial carcinoma. In this study, the authors highlight that obturator vein lymph node might be the most common metastasis site of pelvic lymph node in endometrial carcinoma patients. Understanding the lymphatic drainage of cancers and the risk factors to lymph node metastasis is important to enable less invasive procedures, decreasing postoperative complications. Even though I have some concerns about their data.

The authors describe the inclusion criteria were patients who underwent total hysterectomy, bilateral salpingo-oophorectomy, and systemic lymphadenectomy with or without paraaortic lymphadenectomy. How many patients were submitted to paraaortic lymphadenectomy? What was their surgical indication to the lymphadenectomy extension? Furthermore, Table 1 shows only lymph nodal metastasis, without differentiating pelvic from paraaortic metastasis. How many paraaortic lymph nodes had metastasis? Also, the percentage of simultaneous pelvic and paraaortic metastasis was not informed.

Moreover, they informed that patients with incomplete systemic or selective lymphadenectomy were excluded. It is important to know how many patients they excluded from their analysis during the period. Also, what was considered incomplete systemic or selective lymphadenectomy? Sentinel lymph node? These could bias their data.

The authors described that a logistic regression was performed. Even though they did not inform risk factors included in the analysis or their results. They should consider a logistic regression with multivariate Cox analysis. The information could enhance their study, identifying patients with higher risk of lymph node metastasis.

Other data presented was an average number of lymph node harvest: 31.5 per case. I think the data that they present may be their median. I would like to know the average number of lymph node they harvest instead of the median.

In conclusion, the initial scientific assumption that obturator vein lymph node might be the most common metastasis site of pelvic lymph node with endometrial carcinoma patients cannot be defined by their analysis.

\section{Reference}

1. $\mathrm{Hu}$ J, Zhu W, Wen H (2020) A retrospective mapping of pelvic lymph node metastasis distribution in patients with endometrial carcinoma. Indian J Surg 4:125

Publisher's Note Springer Nature remains neutral with regard to jurisdictional claims in published maps and institutional affiliations.

Ricardo Pedrini Cruz ricardoc@ghc.com.br

1 Department of Gynecology Oncology, Hospital Nossa Senhora da Conceição, Av. Francisco Trein, 596, Porto Alegre, RS 91350-20, Brazil 